POS PROCEEDINGS

\title{
Measurements and interpretations of Simplified Template Cross Sections, differential and fiducial cross sections in Higgs-boson decays to two photons with the ATLAS detector
}

\author{
Eleonora Rossi ${ }^{\star}$ on behalf of the ATLAS Collaboration \\ LAPP, Univ. Savoie Mont Blanc, CNRS/IN2P3, Annecy, France \\ E-mail: eleonora.rossi@cern.ch
}

\begin{abstract}
Higgs-boson decays to two photons can be selected with high efficiency and the very good invariant mass resolution allows a robust subtraction of the backgrounds. This proceeding presents measurements of Simplified Template Cross Sections, differential and fiducial cross sections, as measured in the diphoton decay channel by the ATLAS detector using the full Run 2 data set of proton-proton collision data collected at $13 \mathrm{TeV}$ at the LHC, and their interpretations in the context of an Effective Field Theory.
\end{abstract}

\footnotetext{
*** The European Physical Society Conference on High Energy Physics (EPS-HEP2021), *** ***26-30 July $2021 * * *$

*** Online conference, jointly organized by Universität Hamburg and the research center DESY ***
} 


\section{Introduction}

Measurements in the diphoton final state are really crucial to explore the properties of the Higgs boson and to provide constraints on physics beyond the Standard Model, thanks to the excellent performance of photon reconstruction and identification with the ATLAS detector [1]. The experimental signature of the Higgs decaying into two photons is a narrow resonance with a width consistent with detector resolution rising above a smooth background in the diphoton invariant mass $\left(m_{\gamma \gamma}\right)$ distribution; results are extracted fitting $\left(m_{\gamma \gamma}\right)$ by means of parameterised signal and background shapes.

Two complementary approaches are explored, i.e. measurements of fiducial cross sections and of simplified template cross sections (STXS) [2,3] of the Higgs boson decaying into two photons using the full Run-2 proton-proton collision data set $\left(139 \mathrm{fb}^{-1}\right)$ collected at a center-of-mass energy $\sqrt{s}=13 \mathrm{TeV}$ by the ATLAS detector [1] during the period 2015-2018.

The first set of measurements represents largely model-independent measurements, inclusive in production mode and including information on the decay, with a fiducial selection that matches experimental selection in order to reduce the full phase space extrapolation and with the results that are generally unfolded, in order to provide measurements independent from the detector resolution or selection efficiencies.

The latter instead, measures the production cross sections in the Stage 1.2 STXS fiducial regions, which are defined at the particle level and in the range $\left|y_{H}\right|<2.5$ of the Higgs-boson rapidity $\left(y_{H}\right)$, helping to reduce theory systematics but being more model-dependent; the Higgs-boson production phase space in its rapidity is partitioned by production process as well as kinematic and event properties targets phase space regions within production modes, exploiting bins of true Monte Carlo quantities like $p_{T}^{H}, N_{\text {jets }}$, and using the SM kinematics as a template.

Interpretations of the differential measurements in the context of an Effective Field Theory (EFT) are also addressed.

\section{Measurement of differential cross sections}

Measurements of differential cross sections are performed in fiducial volumes defined at particle level, that resemble the ATLAS detector acceptance and analysis selections.

The $p_{T}^{\gamma \gamma}$ distribution, shown in Figure 1 (a), is compared to NNLOJET+SCET [6] which provides predictions using a next-to-next-to-next-to-leading logarithmic (N3LL) resummation matched to a next-to-next-to-leading order (NNLO) fixed-order calculation in the heavy top-quark mass limit. The distribution reaches out to $350 \mathrm{GeV}$, a region where top-quark mass effects start to become sizeable; on the lower $p_{T}^{\gamma \gamma}$ spectrum, in order to probe the region where resummation effects are important and to probe the charm-quark Yukawa coupling, a fine binning has been chosen. Agreement is observed between the measured $p_{T}^{\gamma \gamma}$ distribution and the default SM prediction ( $\mathrm{p}$-value of $44 \%)$.

Cross sections have been measured also as a function of different jet kinematic observables with sensitivity to new physics, compared to the state-of-the-art theory predictions and used for the interpretations. The distribution of the azimuthal angular difference of the two leading jets, $\Delta \phi_{j j}$, which has sensitivity to the CP properties of the Higgs boson, is compared to Sherpa (Meps@ Nlo) [7-10] 
and GoSam [11, 12] that are of NLO accuracy for this jet multiplicity, as shown in Figure 1 (b). Agreement is observed between this distribution and the expected shape in the SM (p-value of $82 \%)$ [4].

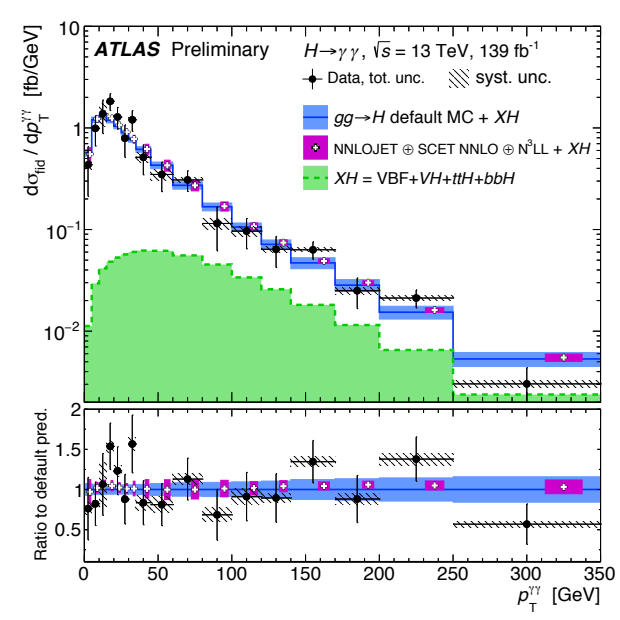

(a)

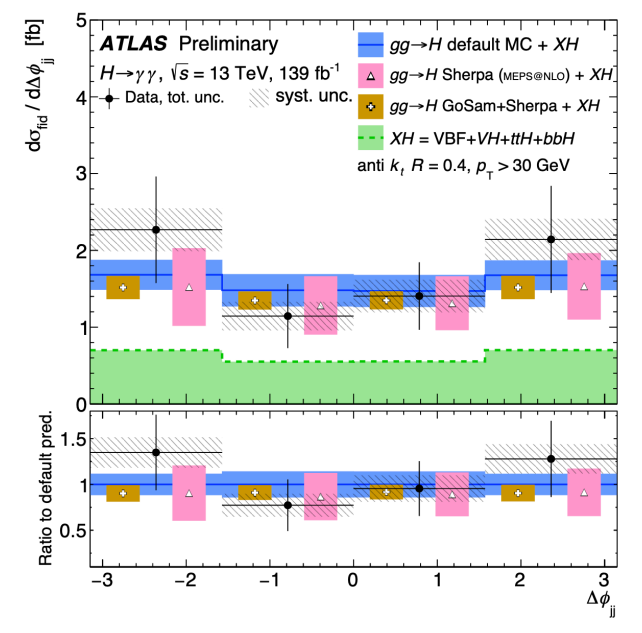

(b)

Figure 1: Cross sections measured as a function of the diphoton $p_{T}^{\gamma \gamma}$ (a) and of a jet kinematic observable, $\Delta \phi_{j j}$ (b) [4].

\section{Measurements of cross sections separated by production modes and in STXS regions}

The Higgs-boson production is firstly probed by considering separately the different production modes, i.e. gluon-gluon fusion $(g g F)$, vector boson fusion $(V B F)$, associated production with a $W$ or $Z$ boson ( $W H$ and $Z H-V H$ when $W=Z=V$ ) and the top-associated production processes. The measurements are reported in Figure 2 in terms of the products of the cross sections times branching fraction, $\sigma \times B R_{\gamma \gamma}$, normalised to their SM predictions, being the cross sections defined in $\left|y_{H}\right|<2.5$.

The leading systematic theoretical uncertainty arises from the modelling of parton shower, while the dominant experimental systematic uncertainty arises from the modelling of background $m_{\gamma \gamma}$ distribution and from the measurement of jets, when considering the $g g F$ and $V H$ categories or the $V B F$ and $t \bar{t} H$ processes, respectively.

The CLs method [13] is used to set an upper limit of eight times the SM prediction for the $t H$ production process.

The Higgs-boson production has been further characterised by exploiting cross-section measurements in the STXS framework.

Results are presented in terms of a set of merged STXS regions defined in each production process, i.e. 27 regions of the Higgs-boson production phase space, and are shown in Figure 3 (a) along with the corresponding correlation matrix in Figure 3 (b). The relative uncertainties on the measurements range from $20 \%$ to more than $100 \%$, with larger uncertainties occurring in particular in regions of high $p_{T}^{H}$ and $p_{T}^{V}$ as well as the low- $m_{j j}$ regions of $q q^{\prime} \rightarrow H q q^{\prime}$. 


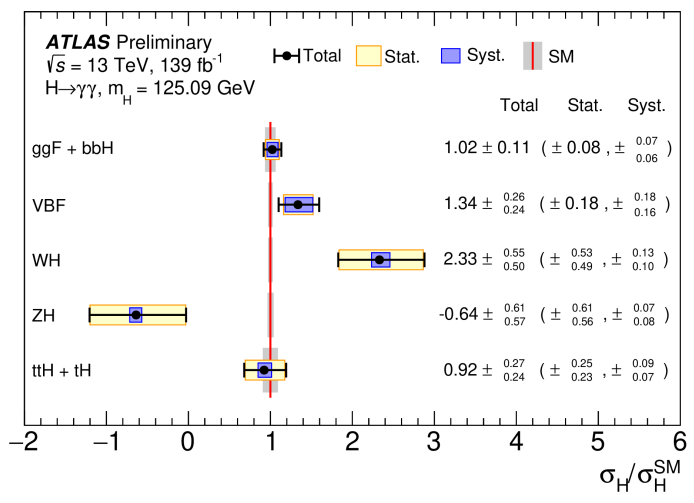

Figure 2: Cross sections times branching fraction for $g g F+b \bar{b} H, V B F, V H$ and $t \bar{t} H+t H$ production, normalised to their SM predictions. The values are obtained from a simultaneous fit to all categories [5].

The systematic component of the uncertainties is smaller than the statistical component in most of the cases while it has similar values for the 0 -jet region of $g g \rightarrow H$.

No significant deviations from the SM expectation have been observed; the compatibility between the measurements and the SM predictions corresponds to a p-value of $60 \%$ [5].

\section{EFT interpretations of differential cross sections}

An effective field theory approach applied to the differential cross section measurements can be used to probe the presence of physics beyond the SM in a model-independent way. In this approach, the SM Lagrangian is supplemented by additional dimension- 6 operators, while the dimension- 5 and dimension-7 operators are excluded assuming lepton and baryon number conservation and the dimension-8 and higher neglected for further suppression:

$$
\mathcal{L}_{E F T}=\mathcal{L}_{S M}+\sum_{i} \frac{c_{i}}{\Lambda^{2}} O_{i}^{(6)}
$$

where the $c_{i}$ are called the Wilson coefficients (WC) and are the free parameters of the theory specifying the strength of the new interactions, while $\Lambda$ is the scale of new physics.

Among the available bases used to parameterise the dimension- 6 operators, the following ones have been employed:

- the SILH basis of the Higgs Effective Lagrangian [14] (SILH);

- the Warsaw basis of the SMEFT Lagrangian $[15,16]$ (SMEFT).

Additional CP-even and CP-odd interactions can change the event rates, the kinematic properties of the Higgs boson, the overall production cross section, from those predicted by the SM.

In this report, the sensitivity of five differential $H \rightarrow \gamma \gamma$ cross sections as a function of $p_{T}^{\gamma \gamma}, N_{j e t s}$, $p_{T}^{j 1}, m_{j j}$ and $\Delta \phi_{j j}$, to operators that affect the Higgs-boson interactions with gauge bosons is explored, while new interactions between the Higgs boson and fermions would impact the inclusive production cross-section directly via the ggF mode. 


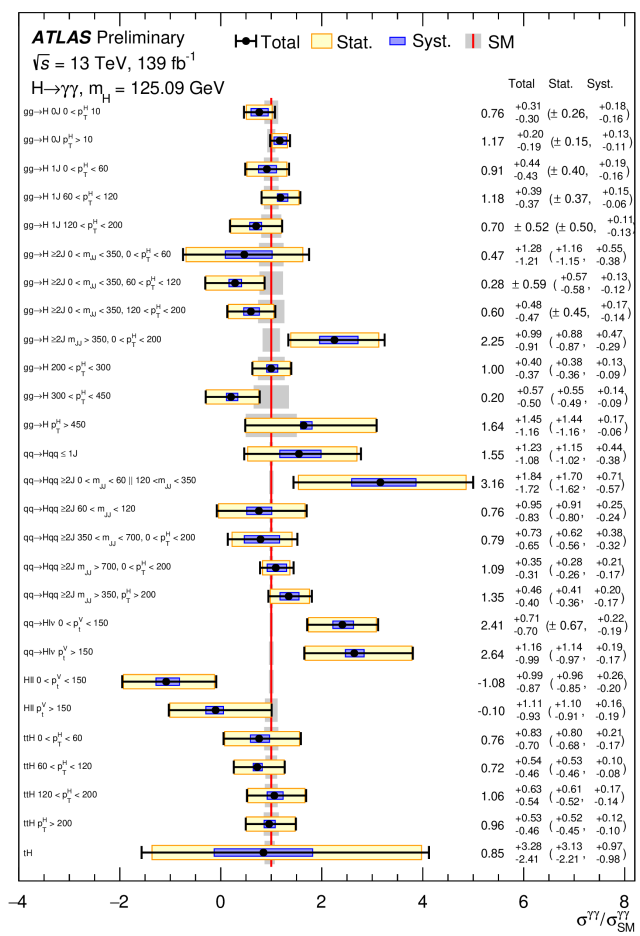

(a)

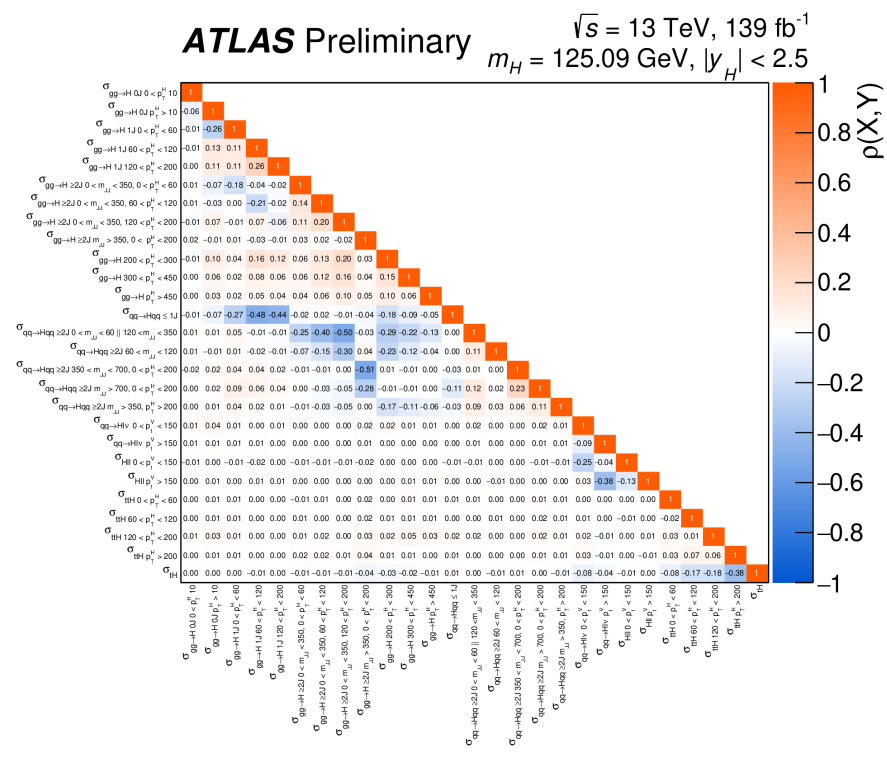

(b)

Figure 3: Best-fit values and uncertainties for the cross sections in each STXS region, normalised to the SM predictions for the various parameters. The values for the $g g \rightarrow H$ process also include the contributions from $b \bar{b} H$ production (a). Correlation matrix for the STXS measurement (b) [5].

Limits on the WC are set by building a likelihood function exploiting the measured and predicted differential cross sections of the five aforementioned observables and the total covariance matrix defined by the sum of the statistical, systematic and theoretical covariances.

A simultaneous fit has been performed in order to constrain at the same time the $\bar{c}_{H W}$ and $\tilde{c}_{H W}$ in Figure 4 (a) $\left(\bar{c}_{H B}=\bar{c}_{H W}\right.$ and $\left.\bar{c}_{H B}=\bar{c}_{H W}\right)$ and the $\bar{c}_{g}$ and $\tilde{c}_{g}$ Wilson coefficients of the SILH basis in Figure 4 (b), setting all the other WC to 0 . These operators introduce new HWW, HZZ and $H Z \gamma$ interactions and new interactions between the Higgs boson and two gluons, respectively. The structure seen in the observed limits in Figure 4 (b) in the two-dimensional parameter plane comes from the destructive interference which causes the ggF production cross section to be zero around $\bar{c}_{g} \sim-2.2 \cdot 10^{-4}$ for $\tilde{c}_{g} \sim 0$. Additional constraints on a set of SMEFT WC, determining the strength of operators that affect the $g g F, V B F$ and $V H$ production and the Higgs-boson decay to photons, are extracted and reported in Figure 5 showing $68 \%$ and $95 \%$ CL limits using the interference between the SM and the dimension-6 SMEFT operators. No significant new physics contributions are observed.

\section{Conclusion}

Two complementary approaches have been exploited in order to measure the Higgs cross sections in the diphoton decay channel. All the results have been proved to be in agreement with the 


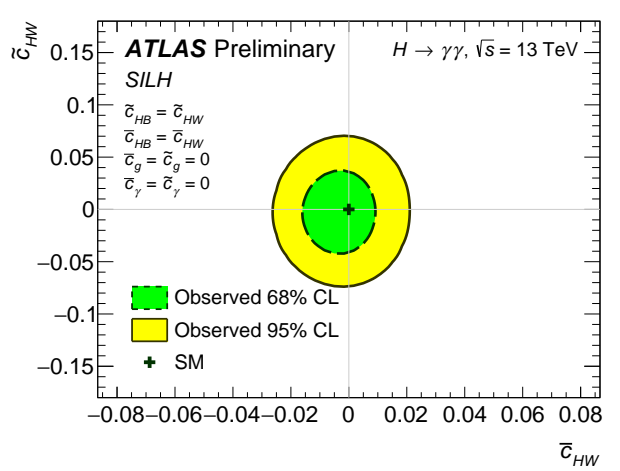

(a)

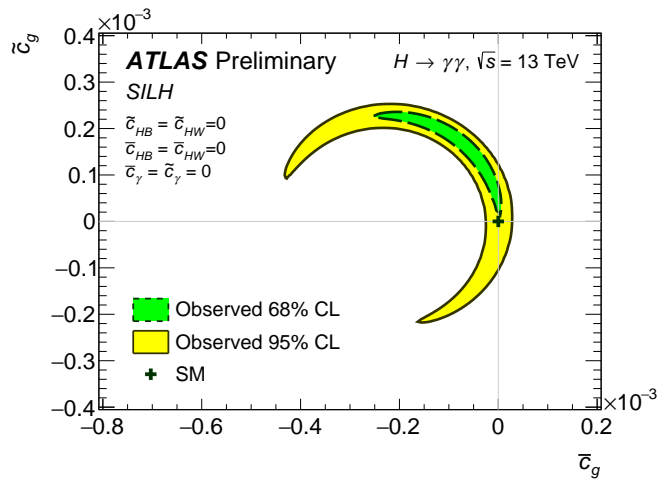

(b)

Figure 4: Observed 68\% (green) and 95\% (yellow) confidence level regions obtained fitting at the same time $\bar{c}_{H W}$ and $\tilde{c}_{H W} ; \bar{c}_{H B}$ and $\tilde{c}_{H B}$ are set to be equal to $\bar{c}_{H W}$ and $\tilde{c}_{H W}$, respectively, while all other Wilson coefficients are set to zero (a); corresponding limits from the simultaneous fit to $\bar{c}_{g}$ and $\tilde{c}_{g}$, setting all other Wilson coefficients to zero (b) [4].

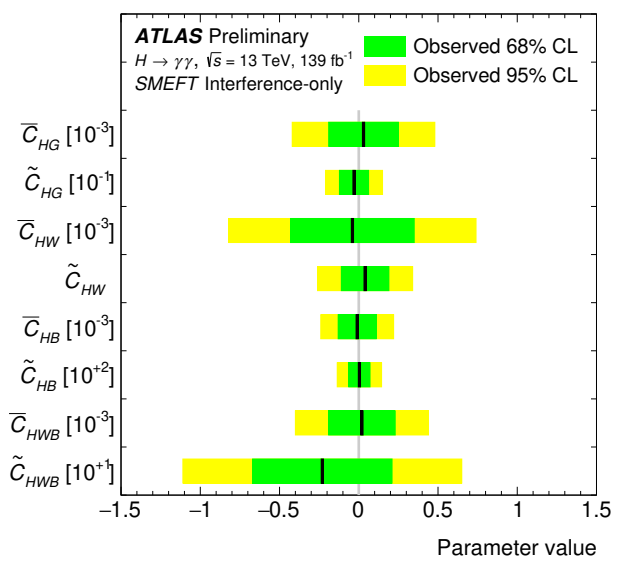

Figure 5: Observed 68\% and 95\% CL limits on SMEFT Wilson coefficients. Limits are derived fitting one Wilson coefficient at a time while setting the other coefficients to zero [4].

Standard Model predictions.

An upper limit of 8 times the SM prediction has been set on $t H$ production. STXS cross section measurements have been provided in 27 regions of Higgs-boson production phase space.

Fiducial differential results have been interpreted in the context of EFT theories in order to constrain the strength of anomalous interactions.

\section{Acknowledgments}

The author's work has been funded by LabEx ENIGMASS. 


\section{References}

[1] ATLAS Collaboration: The ATLAS Experiment at the CERN Large Hadron Collider, JINST3 S08003, 2008;

[2] D. de Florian et al., Handbook of LHC Higgs Cross Sections: 4. Deciphering the Nature of the Higgs Sector, (2016), arXiv: 1610.07922 [hep-ph].

[3] N. Berger et al., Simplified Template Cross Sections - Stage 1.1, (2019), arXiv: 1906.02754 [hep-ph].

[4] ATLAS Collaboration, Measurements and interpretations of Higgs-boson fiducial cross sections in the diphoton decay channel using $139 \mathrm{fb}^{-1}$ of pp collision data at $\sqrt{\mathrm{s}}=13 \mathrm{TeV}$ with the ATLAS detector, ATLAS-COM-CONF-2019-046;

[5] ATLAS Collaboration, Measurement of the properties of Higgs boson production at $\sqrt{s}=13$ $\mathrm{TeV}$ in the $H \rightarrow \gamma \gamma$ channel using $139 \mathrm{fb}^{-1}$ of p p collision data with the ATLAS experiment, ATLAS-COM-CONF-2020-035;

[6] X. Chen et al., Precise QCD Description of the Higgs Boson Transverse Momentum Spectrum, Phys. Lett. B 788 (2019) 425, arXiv: 1805.00736 [hep-ph];

[7] Bothmann et al., Event Generation with Sherpa 2.2, (2019), arXiv: 1905.09127 [hep-ph].

[8] S. Höche, F. Krauss, M. Schönherr and F. Siegert, QCD matrix elements + parton showers: The NLO case, JHEP 04 (2013) 027, arXiv: 1207.5030 [hep-ph];

[9] S. Höche, F. Krauss and M. Schönherr, Uncertainties in MEPS@NLO calculations of $h+j e t s$, Phys. Rev. D 90 (2014) 014012,arXiv: 1401.7971 [hep-ph];

[10] M. Buschmann et al., Mass Effects in the Higgs-Gluon Coupling: Boosted vs Off-Shell Production, JHEP 02 (2015) 038, arXiv: 1410.5806 [hep-ph];

[11] G. Cullen et al., Automated one-loop balculations with GoSam, Eur. Phys. J. C 72 (2012) 1889, arXiv: 1111.2034 [hep-ph];

[12] G. Cullen et al., GOSAM-2.0: a tool for automated one-loop calculations within the Standard Model and beyond, Eur. Phys. J. C 74 (2014) 3001, arXiv: 1404.7096 [hep-ph];

[13] A. L. Read, Presentation of search results: the CLS technique, J. Phys. G 28 (2002) 2693;

[14] G. F. Giudice, C. Grojean, A. Pomarol and R. Rattazzi, The strongly-interacting light Higgs, JHEP 06 (2007) 045, arXiv: 0703164 [hep-ph];

[15] I. Brivio and M. Trott, The Standard Model as an Effective Field Theory, Phys. Rept. 793 (2019) 1, arXiv: 1706.08945 [hep-ph];

[16] B. Grzadkowski, M. Iskrzynski, M. Misiak and J. Rosiek, Dimension-Six Terms in the Standard Model Lagrangian, JHEP 10 (2010) 085, arXiv: 1008.4884 [hep-ph]. 